\title{
THE OBJECTIVITY OF TRUTH, MORALITY, AND BEAUTY
}

\author{
STEVEN JAMES BARTLETT
}

\begin{abstract}
Whether truth, morality, and beauty have an objective basis has been a perennial question for philosophy, ethics, and aesthetics, while for a great many relativists and skeptics it poses a problem without a solution. In this essay, the author proposes an innovative approach that shows how cognitive intelligence, moral intelligence, and aesthetic intelligence provide the basis needed for objective judgments about truth, morality, and beauty.
\end{abstract}

The author has chosen to issue this essay as a free open access publication under the terms of the Creative Commons Attribution-NonCommercial-NoDerivs license, which allows anyone to distribute this work without changes to its content, provided that both the author and the original URL from which this work was obtained are mentioned, that the contents of this work are not used for commercial purposes or profit, and that this work will not be used without the author's or his executor's permission in derivative works (i.e., you may not alter, transform, or build upon this work without such permission). The full legal statement of this license may be found at

http://creativecommons.org/licenses/by-nc-nd/4.0/legalcode

(c) $(1) \odot$

(C) 2017 Steven James Bartlett 


\title{
THE OBJECTIVITY OF TRUTH, MORALITY, AND BEAUTY
}

\author{
STEVEN JAMES BARTLETT
}

his famous Trio_-Truth, Morality (or the Good), and Beauty — was firmly
set at the very center of Western philosophical thought ever since Plato
wrote about them, and there it has remained, subject to the vagaries of changing philosophical fashion and style, vested interests, and preferred paradigms. For more than two millennia, the same fundamental question has haunted the members of the Trio, Is objectivity in connection with any one or more of them possible, and if so, can such objectivity be established so as to satisfy the standards of rational thought?

As the formal science of mathematics has developed, and somewhat less so as physical science has reached periods of great confidence in its results, only to be revised and recast as a consequence of conceptual revolutions, objective Truth has seemed more readily approachable than has objectivity in connection with Beauty or Morality.

And yet, not even as the most secure and demonstrable of all areas of human knowledge, mathematics, too, has been forced to take steps backwards or sideways, as it has been compelled by its own findings to recognize forms of intellectually humbling internal theoretical limitation. Some of these limitative proofs were developed by Gödel, whose work has become best known, but results by a succession of other mathematicians-including Kleene, Rosser, Kalmár, Gentzen, Church, Turing, Post, Tarski, Mostowski, Löwenheim, Skolem, Henkin, Wang, and others-have extended, amplified, modified, and in a variety of ways and, to a degree, have even found ways to circumvent some internal limitations of 
mathematics. Their contributions, developed from differing formalized perspectives, point in the direction of what I have termed "framework-relativity," which recognizes that all claims to knowledge, mathematical or otherwise-including the claim I am making here-are intimately tied to, and limited by, the conceptual frameworks that make those claims possible.

The same pattern of development has been witnessed in physics, where special and general relativity recognize the inseparable connection of physical results from the reference frames in terms of which physical laws are expressible and are subject to confirmation or disconfirmation. Quantum theory, in a similar way, is based on the parallel recognition of essential and unbreakable ties among the physically observable, the physical observer, and the theoretical framework presupposed in order to interrelate the preceding two.

These theoretically intimate, essential, nonseverable relationships of connection between the objects measured by physics and the theoretical frameworks that are presupposed by agent-bound, measurement-bound descriptions of physical regularities that we can come to recognize, all tell very much the same theoretical story as in mathematics: that whatever we can know, whatever we are capable of formulating and communicating, whatever we are able to prove, all of these rely for their very possibility and meaning upon the frames of reference that we cannot help but presuppose in the process.

Framework-relativity is, however, not "relativism," a now-widespread popular presumption that claims-no matter what purported "truths" are discovered or believed to be proved-that such "truths" are no more than provisional, tentative, and dismissible whenever we feel like shrugging them off, since all we need to do is remind ourselves that "everything really is subjective," that "nothing can be known objectively to be true," etc. There are, of course, good, strong, and rationally convincing arguments against relativism, the best of which, in my view, points to the fact that the relativist's own position cannot possibly be articulated without 
presuming the very objectivity that he or she wishes to deny. But here I am not concerned to refute relativism, only to dismiss it as irrelevant to the concept of framework-relativity, which, contrary to relativism, recognizes the objectivity of results relative to the frameworks those results presuppose.

This is not a sophism, not a smokescreen to disguise, elevate, and dignify the "merely relative" by coining an unfamiliar phrase. Let me give some examples of objective results that are framework-relative: Euclidean geometry's theorems are objective in this sense; they are provably true relative to a set of definitions, postulates, and rules of inference. Similarly, Riemannian geometry, which rejects Euclid's parallel postulate, leads to a set of true propositions, which are provable relative, again, to a specified set of definitions, postulates, and rules of inference. Lobashevskian geometry, which also repudiates the parallel postulate in a different way, leads to demonstrable results relative to an alternative set of definitions, postulates, and rules of inference.

All such results are objectively demonstrable. What meaning does the term 'objectivity' have, other than simply the capacity to establish results that are not subject to controversy and can be replicated by competent colleagues ${ }^{1}$ in a given discipline_-provided that they accept the framework which the establishment of such results must presuppose? If they don't accept that framework, they beg the question, they engage in what philosophers call a petitio principii-they refuse to abide by the rules of the game at issue, perhaps because they wish to use alternative rules and play a different game, or perhaps because they are being intellectually recalcitrant, believing that the skepticism they express is not itself self-undermining (which it is). ${ }^{2}$

As long as you accept the rules of chess, some moves are objectively good, and others are objectively bad. As long as you accept the definitions, postulates, and rules of

\footnotetext{
1 As we shall see later on, such "competence" means having in common with colleagues a relevant form of intelligence without which the results in question could not be obtained or adequately understood.

2 See note 3 .
} 
inference of Euclidean geometry, then there are results in Euclidean geometry that are objectively provable. This is what 'framework-relativity' means, and the metatheory that leads to the recognition of framework-relativity is itself objectively provable-by showing that any (possible) attempt to deny it leads to self-referential inconsistency of a particularly devastating kind. ${ }^{3}$

Mathematicians have long been both aware and appreciative of the essential interrelationship between the formal systems they study and the validity of the results those formal systems entail. Physicists have, during the century since Einstein's special and general theories of relativity, come to understand and also appreciate how the conceptual structures in terms of which their observations are both made and then are formulated reveal an underlying logic of framework-relativity. Neither mathematicians nor physicists have, as a result of this growing framework-relative awareness, given up the search for, and claims on behalf of, objectively demonstrable results. This is not because mathematicians and physicists are intellectually stubborn or obtuse, it is because 'objectivity' simply means_and the very possible meaning of the term itself necessarily requires_-framework-relativity.

Let us suppose, for the sake of moving on, that there is a well-defined sense in which mathematics and physics can attain to objectively valid results, objective truths. In admitting this, it is not necessary that we always know for certain that these results are, at the time, objectively valid, but only that, given sufficient time and continuing effort, objective Truth stands as a meaningful goal in mathematics and in physics, a goal that satisfies basic tenets of rationality. (What I generally mean by the last phrase will be made clearer in the context of our later discussion of forms of intelligence).

Let us turn to Morality and Beauty, those more nebulous and less intellectually manageable ideas compared with Truth. Is there any possible sense in which

\footnotetext{
${ }^{3}$ Sample publications by the author that bear this out will be found under "References" at the end of this paper.
} 
propositions of morality or in which those of aesthetics can be "objectively established?"

These two areas, morality (or ethics) and aesthetics (or the theory of beauty), have remained the ultimate refuges of unbridled subjectivity. For these are areas in which-by nearly universal admission by those who are not afflicted by unyielding religious dogma-it is believed that "one person's meat is another's poison," that "my belief is as good as yours," that "thinking makes it so," and so on. In the world of reality, aesthetics therefore has been associated with "personal taste," and morality with "personal moral inclination." Tastes and inclinations are inherently subjective terms and suffer from the aura of vagueness and the arbitrariness of relativism. To go beyond the merely subjective in connection with Beauty and Morality, we will take a path not previously travelled which involves an understanding of two unfamiliar and newly identified varieties of human intelligence.

\section{Major forms of human intelligence}

\section{Cognitive intelligence}

Is human IQ an objectively determinable thing? Are cognitive abilities such as verbal comprehension, problem-solving, pattern recognition, memory, mental processing speed, etc., measurable, and if so, are the conclusions reached on the basis of such measurements in any sense "objective"?

The answer to this question is as non-arbitrary as are the results derived in a system of Euclidean geometry. Once definitions of the relevant cognitive skills are specified, tests are constructed to measure those skills, the tests are then given to sample groups of people, and finally the test results themselves are carefully evaluated to answer the question, Do individuals whose test scores are high (or low) reliably exhibit the designated skills to a high (or low) degree? Additional, alternative/supplementary ways to assess the skills being tested may then be brought in to confirm the test results. Once a psychometric test has been validated in these 
ways with large populations, to ask whether the test results are "objective" is to ask one of three questions: whether the definitions of the relevant skills are to be accepted, or whether the test that has been constructed actually succeeds in measuring those skills effectively, or sometimes a third and misconceived question is asked, to which we'll come in a moment.

The second question is of course more easily answered: We look for additional means to strengthen the IQ test validation process. There are numerous ways to do this: personal interviews with the test subjects, data about their cognitive success rates in real life, etc. It is the first question that often misleads people, especially those who believe that definitions are somehow dictated by reality, and, because they believe this, they disagree about what "real intelligence" actually is. ${ }^{4}$

Reality, whether we may be concerned to designate specific cognitive skills, or whether we may want, for example, to specify the features that define and differentiate biological species, does not come pre-classified or precompartmentalized. It is rather our own interests and goals that lead us to regard certain cognitive skills, or certain biological characteristics in the case of species, to be significant, important, or relevant, and we then formulate definitions that reflect those interests and goals. ${ }^{5}$

We value such cognitive skills as verbal abilities, spatial relations skills, the ability to calculate, the ability to remember, the ability to comprehend, to imagine, to think critically. These cognitive skills reflect basic human interests and goals, and those interests and goals motivate us to designate these and related skills as important. IQ tests are simply constructed to test for the skills that we choose to define as cognitively important because they reflect our vested interests and goals. These interests and goals establish a framework relative to which we have developed ways

\footnotetext{
${ }^{4}$ On the kinds of definition and the belief in "real definitions," see Bartlett (2011, Chap. 2).

${ }^{5}$ On the so-called species problem in biological taxonomy, see Bartlett (2015).
} 
of testing individuals for the presence of those skills which our interests and goals lead us to recognize as "cognitive intelligence skills."

But there is a third question we need to mention, one that the IQ skeptic insists upon asking about IQ tests: whether people whose test results show them to have high (or low) IQs really are cognitively intelligent (or lacking such intelligence). To raise this question is again to engage in a petitio principii- to refuse to accept a set of rules that establishes a system that identifies certain skills as manifestations of "cognitive intelligence" as it has been defined by IQ tests. Either the skeptic is exercising his or her "right" to be intellectually uncooperative (wanting to play "chess" but refusing to accept the rules of chess), or underlying the question is a wish to assert the skeptic's "right" to specify an alternative, broader, or narrower set of defined skills, and by so doing to formulate an alternative, broader, or narrower conception of "intelligence."

That latter "right" is always assured, and it has in fact been exercised by investigators of intelligence so as to produce conceptions of intelligence that are distinguishable from the standard skills associated with cognitive intelligence. The study of human intelligence at this time recognizes a variety of forms of intelligence, among them, emotional intelligence, ${ }^{6}$ social intelligence, ${ }^{7}$ as well as other varieties, and more recently in the author's research, moral intelligence ${ }^{8}$ and epistemological intelligence. ${ }^{9}$

The multiplicity of kinds of human intelligence does not imply that any one of them is any the less amenable to objective measurement merely because other varieties have also been identified. The same situation exists, as we have seen, in connection with the family of alternative geometries. The existence of conceptual alternatives does in itself not stand in the way of objectivity. Relative to each

\footnotetext{
${ }^{6}$ Among the earliest authors, see, e.g., Salovey \& Mayer (1990), Gardner (1993, 1993/1983), Goleman (1995).

${ }^{7}$ The historical development of the concept of social intelligence can be found in going from one of these publications to the next: Thorndike (1920), Spearman (1927), Bruner \& Tagiuri (1954), Taft (1956), Guilford (1967), Eysenck (1999).

${ }^{8}$ Bartlett (2002), Bartlett (2005, Chap. 18) and (2011, Chap. 1).

${ }^{9}$ Bartlett (2017a).
} 
reference frame that clearly specifies what is to be identified and how, we are able to recognize and reach public agreement about whatever objects that frame of reference enables us to designate. This is most fundamentally what the term 'objectivity' means.

\section{Moral intelligence}

We now shift our attention from cognitive intelligence to what I have elsewhere called "moral intelligence." ${ }^{10}$ Like the cognitive variety, moral intelligence is defined in terms of a set of skills. - Once again it is important to be reminded, reality does not dictate the specific skills we select, but rather our goals and interests do; they determine what we value. The fact that values are basic and central to any concept of intelligence, of whatever variety, does not imply that we are therefore automatically dealing with "purely subjective," "arbitrary," or "vague” ideas.

Framework-relativity in connection with any given variety of intelligence means two things: (i) that a certain set of values is necessarily presupposed, values that underlie the interests and goals which the given form of intelligence manifests, and (ii) that it is relative to those values that the given variety of intelligence possesses the meaning we ascribe to it. If we make an effort to define clearly-in a manner that permits objective validation-the set of skills that we wish to recognize, then the resulting concept of intelligence that we formulate becomes capable of objective reference.

The concept of moral intelligence can be defined in terms of a set of specific skills and dispositions (how an individual will likely behave). The skills and dispositions we select in defining moral intelligence is not arbitrary. In the case of cognitive intelligence, we look to human models_-human exemplars_of high cognitive intelligence in order to direct attention to skills that we value in cognitively high-functioning individuals. In the case of moral intelligence, we do the same thing:

\footnotetext{
10 See note 8 , also Bartlett (2017a).
} 
we look to human individuals whom we judge to exemplify high-functioning moral reasoning and behavior.

Just as we have a traditional consensus that cognitively high-functioning individuals are to be found in connection with intellectually demanding high achievement, morally high-functioning individuals are found among outstanding spiritual leaders and great humanitarians; in addition, we also recognize highfunctioning moral reasoning and behavior in those less celebrated, more ordinary people who dedicate themselves to human welfare: fighters against injustice, cruelty, bias, prejudice, and persecution, those who struggle and sacrifice to help the unfortunate, etc. There are many ways in which moral intelligence can be manifested. Individuals whom we identify as morally high-functioning serve as a "reference group" in relation to which it is not a difficult matter to identify the dispositions and skills that characterize their way of thinking and of behaving.

Among the main dispositions and skills that I have identified in studying moral intelligence are these: a strong psychological aversion to participation in behavior that harms others, a fully functioning capacity for empathy, a well-developed sensibility that is expressed in normative weighing tied to aversion that a morally intelligent person feels when confronted by typical human aggression and destructiveness, and a strong moral conviction that bridges skills in moral reasoning with commitment to act accordingly. ${ }^{11}$

Here in this short essay it is not my intent to describe in detail and to discuss the dispositions and skills related to moral intelligence, but rather to point to the fact that, once we have clearly formulated definitions of those dispositions and skills in hand, along with criteria that inform us how we can recognize those dispositions and skills in people, we then have at our disposal a frame of reference in terms of which

\footnotetext{
${ }^{11}$ For detailed discussions of the dispositions and skills that constitute moral intelligence, see notes 8 and 10 .
} 
it is possible to identify morally intelligent individuals. And once that reference frame is established, "moral intelligence" becomes objectively identifiable.

Once morally intelligent individuals are objectively identifiable, it is then a short step to identify "objective moral behavior," which, it follows, is the behavior that morally intelligent people engage in.

Treatise-upon-treatise has been written about morality, attempting-by hook or by crook! - somehow to justify moral admonitions, and books-upon-books about ethics have urged reasons for the adoption of ethical principles. These works fill many of the shelves of libraries. Moral and ethical discussions over millennia have been interminable, and they have led to no objectively establishable conclusions. They have led to dogmas and doctrines, to pronouncements, and to often bitter controversy.

It is, however, possible to provide a clear and persuasive response to the question, Does morality have objective validity? A clearly formulated concept of moral intelligence provides a natural and empirically-based means of understanding and conceptualizing moral reasoning and behavior, and it also provides us with a psychologically-based comprehension of why some individuals behave morally and others do not. ${ }^{12}$

\section{Aesthetic intelligence}

Of the members of the illustrious Trio, least amenable to objective validation has very likely been aesthetic judgment concerning Beauty. What constitutes Beauty and how is it to be recognized? Are some works of art "objectively beautiful," or is beauty merely in the eye of the beholder, an entirely subjective affair? Is there any set of standards or criteria by means of which high aesthetic quality (and low) can be objectively recognized?

\footnotetext{
12 Bartlett (2005 and 2011, Chap. 1 and passim.)
} 
Again, the shelves of libraries are packed with treatises and disquisitions that attempt to answer these questions. I think it is fair to say that none has.

An untried answer to the question follows readily from a recognition of a largely overlooked variety of human intelligence which it is appropriate to call "aesthetic intelligence." As we shall see, once we gain an understanding of aesthetic intelligence, an acknowledgment of framework-relativity leads in a straightforward way to the objectivity of Beauty, as it does in the cases of Truth and Morality.

One of the most outspoken, articulate, and scientifically-oriented researchers in the field of aesthetics has been Charles Murray. His carefully compiled study, Human Accomplishment: The Pursuit of Excellence in the Arts and Sciences, 800 B.C. to 1950, takes a stand on behalf of the objectivity of scientific Truth as well as the objectivity of Beauty:

Judgment is separable from opinion in matters of artistic and scientific excellence. It is possible to distinguish the important from the trivial, the fine from the coarse, the credible from the meretricious, and the elegant from the vulgar. Doing so is not a simple matter, and no single observer is infallible, but a realm of objective knowledge about excellence exists. That knowledge can be tapped systematically and arranged as data that meet scientific standards of reliability and validity. (Murray, 2003, p. xvi)

Murray was certainly aware of objections to his claim of aesthetic objectivity. Among the main objections is the flat-out opposition to the very notion that aesthetic judgments can be objective. There is, he realized, ". . . hostility to the idea that discriminating judgments are appropriate in assessing art and literature, or that hierarchies of value exist-hostility as well to the idea that objective truth exists" (p. xviii).

Psychological hostility toward an idea is difficult and usually impossible to reason with; often, in a typically human paradox, the more one attempts to reason with it, the more magnified and intense the opposition becomes. Here, I make no attempt to 
reason with it, but choose to ignore it. Hostility toward an idea has never constituted a valid argument, and rational argument is our interest here, not the psychology of hostility.

In the first passage quoted above from Murray's book, he claims " $a$ realm of objective knowledge about excellence exists." The category of objective knowledge that he has in view includes both scientific and aesthetic knowledge. In connection with the latter, he is concerned with "aesthetic excellence."

Now the usual way in which researchers in aesthetics have approached the matter of aesthetic excellence has been to try to develop criteria or standards in relation to which works of art (here I include music, sculpture, literature, drama, dance, etc.) can be judged on a spectrum from bad to outstanding. This is a reasonable approach to take-to look to the objects of aesthetic creation for relevant standards of excellence. Unfortunately that approach has not been successful in establishing the validity of objectively valid judgments concerning Beauty. The reason for this failure has been the ease with which it is possible to dispute and reject any proposed set of aesthetic standards that apply to created artistic works. There is nothing beyond the works themselves to appeal to that can serve as a basis for objectivity.

It is not so easy, however, to dispute the set of skills that are associated with aesthetic intelligence. ${ }^{13}$ The reason this is the case is due to the existence, again, of a "reference group" in terms of which we can identify the skills that are manifested by aesthetically intelligent individuals. A reference group, one that exists independently of the artistic works about we wish to be able to judge objectively, provides us with a separate basis upon which judgment can be formulated and expressed with detachment. Objectivity of judgment in this context requires just such a separately

13 Of course we are reminded that there is nothing, including even this qualification, that is immune to stubborn disputatiousness. Intellectual recalcitrance can perhaps never be eliminated, but, at the same time, recalcitrance, like hostility, is not a rational position, and rational justification is our only concern here. 
available point de repère-it gives us a place to stand, a locus from which judgment can be made about a subject-matter, from outside that subject-matter.

And yet we see immediately that to appeal to such a reference group presupposes that we are already in possession of implicit criteria that enable us to choose "appropriate" individuals who are to comprise that reference group. This appears to be manifestly circular, does it not? And in fact it is. But not all circular reasoning is "viciously circular."

Sometimes-for logical reasons I will only suggest here and not explain in detail $^{14}$ — we cannot possibly get outside ourselves to gain access to that from which our experience internally limits us. This is not as perplexing as it may at first sound: We cannot experience what lies beyond experience; we cannot think thoughts that we are unable to think; we cannot say the unsayable. In these senses-in our experience, in our thought, and in our ability to express ourselves-we are confined to a closed or recurved universe, whose topology, to speak metaphorically, has the shape of a geometrically closed surface in space (e.g., a Klein surface): No matter how far we travel, we remain in that space; no matter what we think or experience, we remain subject to the limitations of thought or experience.

As inhabitants in such a closed universe, we do not have insurmountable trouble in specifying acceptable criteria in terms of which mathematical or physical truth can be recognized. We have somewhat more trouble specifying acceptable criteria for moral reasoning and conduct, but we can and do accomplish this in the logically circular way in which we can point to recognized spiritual leaders or great humanists and say, "there is an individual with highly developed moral reasoning and conduct."

In the arts, in connection with Beauty, our closed universe is somewhat less helpful because it does not lead us directly, in established and commonly accepted ways, to recognize individuals who possess aesthetic intelligence. But we can

\footnotetext{
${ }^{14}$ For those interested in a more technical, less accessible explanation, see Bartlett (1970) and Bartlett (2017b, forthcoming).
} 
nevertheless begin to formulate the basis for a reference frame in terms of which aesthetic intelligence, and as a result, Beauty, are objectively meaningful.

A descriptive psychology or phenomenology of aesthetic sensibility, discrimination, and judgment has not been formulated in a manner that can be stated clearly in a way similar to that we have found in connection with cognitive and moral intelligence, for there is today no accepted established reference group of aesthetically intelligent leaders. And yet the defining characteristics of aesthetic intelligence are implicit in our consciousness of those whom we would regard as possessing it. Here are some of its main characteristics, which I leave it to the reader to ratify by his or her own cultural experience:

To possess a high degree of aesthetic intelligence is to have, in combination, the following set of skills: the skill of sensitivity to the form, structure, detail, and multilevel meaning of a work of art; the ability to discern levels of meaning, complexity, and the successful integration of these in a work of art; the ability to perceive, appreciate, and discriminate a work's degree of success in communicating its meaning, complexity, and integrity; the ability to recognize and evaluate the artist's degree of skill in realizing the foregoing accomplishments; a capacity to become absorbed in attention and awareness of what the work expresses; the skill of aesthetic discernment, that is, a breadth of consciousness of the gamut of aesthetic possibility open to whatever art form is involved, coupled with a cultivated, informed capacity to recognize the value of a work on a scale of value.

Beyond these skills, there is a further essential characteristic required. It is, we might say, a meta-property of the foregoing skills, for it brings together that set of skills within the unity of a distinguishing perspective. I have described this perspective in some detail in a group of previous publications, ${ }^{15}$ and so here will only state in a few words the property that is central in defining that perspective.

${ }_{15}$ Bartlett (1990, 1993, 1994a, 1994b). 
What unifies the previous set of skills in an individual possessing aesthetic intelligence is a consciousness and deep-seated appreciation of "higher" and "permanent" realities, realities that have a significance which transcends the "lower" and "transient" world of everyday, mundane living. Such higher, permanent realities offer a level of meaning that is perceived by the aesthetically intelligent to be of value in and of itself, and those with aesthetic intelligence immediately recognize this.

Since our society has generally lost touch with such realities, to the point that the previous paragraph can for some readers be mystifying or meaningless, we may wish for a brief reminder of what they are:

To clarify what I mean by 'permanent realities', it will be helpful to mention some of the kinds of frameworks of reference from the standpoint of which permanent realities become evident: Within the traditional liberal arts, . . mathematics, art, music, literature, philosophy, and religion were thought to provide such frameworks of reference. In a wide variety of ways . . . these disciplines focused attention on subjects that mattered to individuals whose interests invested them with certain cares. Generally these cares involved a concern for realities of a higher order [-that is, realities] that do not change with the passage of time, and do not vary in character when identified by different individuals at different times. Terms like 'logos,' 'formal reality,' 'transcendental principles,' etc., were used to refer to them.

These subjects of interest to mathematicians, philosophers, and others were asserted to be perennial in that the permanent realities they study constitute a level of discourse and of understanding that is enduring and beyond the effects of the passage of time. (Bartlett, 1994a, p. 334)

This level of discourse and of understanding was thought to be intellectually elevating: In this sense, the artes liberales liberated the mind from its preoccupation with mundane and fleeting human affairs, bringing the mind into contact with higher concerns that, because they are universal and invariant, occupy a plane of reality higher than that of ordinary and routine daily living. 
In the group of publications I mentioned above, I described an increasingly widespread inability in Western society to perceive reality under the guise of the transformation that "higher values" make possible. The Scholastics called this inability acedia. They associated the word 'cultus'-that is, 'culture'-with the permanent realities that are closed off to those whose capacity to appreciate them is impaired by acedia.

Of course, the word 'culture' is today applied enthusiastically and indiscriminately, to "the culture of a business," "the culture of a sports team," or "the culture of U.S. kindergarten schools." But at one time, the word had a specific unitary significance: It pointed to a level of meaning that is both higher than the commonplace and transient, and provided those with the required sensibility access to permanent realities.

This "required sensibility" is the meta-property of the skills that define "aesthetic intelligence." The concept of aesthetic intelligence did not occur to the Scholastics, nor did it occur to them to spell out in detail what set of skills, and what defining overall perspective, such intelligence involves.

The selection of these defining skills and their meta-property is far from arbitrary, but like the defining skills of cognitive and moral intelligence, the selection made is, in a non-viciously-circular way, dependent upon familiarity with the interests and goals that outstanding aesthetic creation presupposes.

As in the cases of cognitive intelligence and moral intelligence, it is of course possible to specify a variety of competing, alternative interests and goals, and as a function of those to propose alternative conceptions of aesthetic intelligence. But in connection with any variety of intelligence there is a need to take into account the reference group consisting of those who express it to an exceptional degree. With cognitive intelligence, this reference group consists - and this is no accident-of individuals who display a set of recognized cognitive skills to an outstanding degree; the same is true of the reference group consisting of those who exhibit high 
functioning moral thought and conduct. When we consider aesthetic intelligence, we similarly do not proceed in a vacuum, but look to those individuals who possess aesthetic intelligence to an exceptional degree.

The world of art is rich and multivaried; works of art express a huge range of intent and accomplishment, of simplicity and complication, of design or arbitrariness, of success or failure to communicate. The range and diversity of works of art bear within them, much as do the achievements of mathematics and science, and as do expressions of moral thought and behavior, their own requirements of relevant skills both for their accomplishment and for their appreciation.

Aesthetic intelligence is therefore not an empty box that can be filled as one pleases: The skills that are required in the human listener, reader, or spectator are spelled out, implicitly, by artistic creations. The skills of aesthetic sensitivity, discernment, absorption, discrimination, breadth of consciousness, and aesthetic valuation are, in fact, prerequisites laid down by works of art themselves.

I have given aesthetic intelligence more space in this discussion due to the fact that there is today less evident "aesthetic authority" in connection with this form of intelligence in contrast to the very evident "moral authority" that exists and is wellestablished in connection with moral intelligence, and in contrast to the "cognitive authority" represented by acknowledged major thinkers, scientists, and mathematicians who exemplify cognitive intelligence.

The reference group that provides us with a basis for objective aesthetic judgment does, nonetheless, exist, although the size of the population of that group-consisting of those who can be recognized as clear aesthetic authoritieshas diminished in recent decades. That this has happened is an evident consequence of a loss of the sense of fundamental purpose and direction on the part of all of the arts; we get some inkling of this loss by observing the way the term 'art' has come to be applied as indiscriminately as the word 'culture'. 
The phenomenological profile of aesthetic intelligence I have given is intended not as a full and detailed account of the skills that define it, but rather to indicate how it is possible, in relation to a specified set of skills, to recognize the objectivity of Beauty in precisely the same way in which Morality and Truth can be recognized as objective in relation to the skills that define their respective forms of human intelligence.

\section{Intelligence as a Path to Objectivity}

The close relationship between objectivity and intelligence is seldom explored. We take that close relationship for granted when it comes to mathematical and scientific truth; we have, accordingly, developed a well-defined conception of cognitive intelligence as a set of skills which together are essential to the realization and to the understanding of mathematical and scientific truth.

In connection with moral reasoning and moral behavior, the human tendency has been to seek for commandments from above or from beyond human experience, moral injunctions which then are believed to be "objective" and therefore nonarbitrary. As I have tried to prove elsewhere, such recourse to "transcendent reality" is a form of conceptual error, ${ }^{16}$ a primitive wish "to project" beyond the limitations of human experience in order to persuade believers that what they believe is capable of serving as a compelling objective basis for moral goodness or virtue.

The projective wish for transcendent realities has of course brought humanity throughout its history intractable conflicts and great unnecessary suffering. But purely in order to establish the objectivity of morality, the psychological drive to engage in projective belief is unnecessary. In the concept of moral intelligence, we

16 See, for example, Bartlett (2005, Chapter 19, "The Pathology of Everyday Thought": \ "The Delusion of Transcendence and Human Evil"; also 2017a, Appendix: "The Projection of Transcendence"). 
are already in possession of a framework-relative basis for moral objectivity; no more than this is needed.

The same is true in connection with aesthetic intelligence. The skills which define that variety of human intelligence provide the necessary basis for aesthetic judgment that is, in parallel with judgments of cognitive truth and judgments of moral goodness, equally objective.

We have in this essay in a certain sense turned analysis inward to examine the function of "the eye of the beholder" in establishing objectivity. We have found residing behind the beholder's eye three distinct forms of human intelligencecognitive, moral, and aesthetic intelligence-each respectively comprising a pathway to Truth, Morality, and Beauty, and each variety of intelligence providing a basis for the objectivity of its corresponding member of the famous Trio. In the process, we have shifted our interest and attention, from truths, moral precepts, and standards of aesthetics, to the corresponding varieties of human intelligence that enable us to recognize and to develop the objectivity of truth, the objectivity of moral thought and behavior, and the objectivity of beauty-each now no longer spelled with a capital letter, as each now serves our human goals not only as an Ideal, but as an objective reality. 


\section{References}

Bartlett, Steven James (1990). Acedia: The etiology of work-engendered depression. New Ideas in Psychology, 8:3, 389-396.

Bartlett, Steven James (1993). Barbarians at the door: A psychological and historical profile of today's college students. Originally published concurrently in the Netherlands, in Methodology and Science, 26:1, 1993, 18-40, and in the U.S., in Modern Age, 35:4, 1993, 296-310.

Bartlett, Steven James (1994a). The loss of permanent realities: Demoralization of university faculty in the liberal arts. Methodology and Science: Interdisciplinary Journal for the Empirical Study of the Foundations of Science and Their Methodology, 27:1, 25-39

Bartlett, Steven James (1994b). The psychology of faculty demoralization In the liberal arts: Burnout, acedia, and the disintegration of idealism. New Ideas in Psychology, 12:3, 277-289.

Bartlett, Steven James (2002). Roots of human resistance to animal rights: Psychological and conceptual blocks. Animal Law, 8, 143-76. Electronically republished October, 2002, by the Michigan State University's Detroit College of Law, Animal Law Web Center, and maintained on an ongoing basis: http://www.animallaw.info/articles/arussbartlett2002.htm. Translated into German: Wurzeln menschlichen Widerstands gegen Tierrechte: Psychologische und konceptuelle Blockaden. Trans. Gita Y. Arani-May. Electronically published: http://www.simorgh.de/animallaw/bartlett 33-67.pdf. Translated into Portuguese: Raízes da resistência humana aos direitos dos animais: Bloqueios psicológicos e conceituais. Trans. Daniel Braga Lourenço. Brą̧ilian Animal Rights Review (Revista Brasileira de Direito Anima), 2:3, July/December, 2007.

Bartlett, Steven James (2005). The pathology of man: A study of human evil. Springfield, IL: Charles C. Thomas.

Bartlett, Steven James (2011). Normality does not equal mental health: The need to look elsewhere for standards of good psychological health. Santa Barbara, CA: Praeger.

Bartlett, Steven James (2015). The species problem and its logic: Inescapable ambiguity and framework-relativity. Monograph. Published online Fall, 2015, by arXiv.org (http://arxiv.org/abs/1510.01589), in England by CogPrints (http://cogprints.org/9956/), in France by the Centre pour la Communication 
Scientifique Directe's HAL (https://hal.archives-ouvertes.fr/hal-01196519), and in the U.S. by PhilSci (http://philsci-archive.pitt.edu/11655/).

Bartlett, Steven James (2017a). Epistemological intelligence. Monograph, open access publication, available from PhilPapers: https://philpapers.org/rec/BAREI-4 and the Centre pour la Communication Scientifique Directe's HAL: https://hal.archives-ouvertes.fr/hal-01429159.

Bartlett, Steven James (2017b). Horizons of possibility and meaning: The metalogic of reference. Book in progress.

Bruner, J. S. Tagiuri, R. (1954). The perception of people. In Lindzey, G. (ed.), Handbook of social psychology, pp. 634-54. Reading, MA: Addison-Wesley.

Eysenck, Hans J. (1999). Intelligence: A new look. New Brunswick: Transaction.

Gardner, Howard (1993). Multiple intelligences: The theory in practice. New York: BasicBooks.

Gardner, Howard (1993/1983). Frames of mind: The theory of multiple intelligences. New York: BasicBooks. First pub. 1983.

Goleman, Daniel (1995). Emotional intelligence. New York: Bantam.

Guilford, J.P. (1967). The nature of human intelligence. New York: McGraw-Hill.

Murray, Charles (2003). Human Accomplishment: The Pursuit of Excellence in the Arts and Sciences, 800 B.C. to 1950. New York: HarperCollins.

Salovey, Peter \& Mayer, John D. (1990). Emotional intelligence. Imagination, Cognition, and Personality, 9:3, 185-211.

Spearman, Charles E. (1927). The abilities of man. New York: Macmillan.

Taft, R. (1956). Some characteristics of good judges of others. British Journal of Psychology, 47, 19-29.

Thorndike, E.L. (1920). Intelligence and its uses. Harper's Magazine, 140, 227-35. 


\section{About THe Author}

Steven James Bartlett was born in Mexico City and educated in Mexico, the United States, and France. His undergraduate work was at the University of Santa Clara and at Raymond College, an Oxford-style honors college of the University of the Pacific. He received his master's degree from the University of California, Santa Barbara; his doctorate from the Universite de Paris, where his research was directed by Paul Ricoeur; and he has done post-doctoral study in psychology and psychotherapy. He has been the recipient of many honors, awards, grants, scholarships, and fellowships. His research has been supported under contract or grant by the Alliance Française, the American Association for the Advancement of Science, the Center for the Study of Democratic Institutions, the Lilly Endowment, the Max-Planck-Gesellschaft, the National Science Foundation, the Rand Corporation, and others.

Bartlett brings to his research and writing an unusual background consisting of training in clinical psychology, pathology, and epistemology. He is the author and editor of more than 20 books and monographs, and numerous papers and research studies in the fields of psychology, epistemology, and philosophy of science. He has taught at Saint Louis University and the University of Florida, and has held research positions at the Max-Planck-Institute in Starnberg, Germany and at the Center for the Study of Democratic Institutions in Santa Barbara. He is currently Visiting Scholar in Psychology at Willamette University and Senior Research Professor at Oregon State University.

Willamette University hosts a website that provides information about the author and his research, and makes available a large number of his publications in free downloadable from: http://www.willamette.edu/ sbartlet 